\title{
Regulation of Breast Cancer Progression by Phosphorylation of the Tumor Suppressor Tropomyosin-1 Alpha
}

\author{
Mouna Zerradi, François Houle, Jacques Huot* \\ Oncology Division of Laval University Hospital Research Centre (CRCHU of Quebec) and Laval University Cancer \\ Research Centre, L'Hôtel-Dieu of Quebec, Quebec City, Canada \\ Email: Mouna.zerradi.1@ulaval.ca, Francois.Houle@crchuq.ulaval.ca, ․ Jacques.Huot@fmed.ulaval.ca
}

Received 17 July 2015; accepted 25 August 2015; published 28 August 2015

Copyright (C) 2015 by authors and Scientific Research Publishing Inc.

This work is licensed under the Creative Commons Attribution International License (CC BY). http://creativecommons.org/licenses/by/4.0/

(c) (i) Open Access

\begin{abstract}
Background: Tropomyosin 1 alpha chain (Tm1) is an actin-binding protein that regulates the endothelial cell response to oxidative stress following its phosphorylation at Serine 283 (S283). Tm1 is also a major tumor suppressor in breast cancer. In the present study, we investigated the role of phosphorylation of Tm1 in regulating its tumor suppressor properties. Methods: MDA MB231 breast cancer cells stably overexpressing wild type form of Tm1 or Tm1 mutants (S283A and S283E) were generated. Proliferation and cell viability were assayed by means of the enzymatic cleavage of the tetrazolium salt WST-1 to formazan dye by cellular mitochondrial dehydrogenases. Adhesion assays were performed at various periods of time on cells grown on plastic. Cell migration was evaluated by using the wound-healing assay and by measuring transendothelial migration of cancer cells. Malignant transformation in vitro was determined by using the anchorageindependent growth assay on soft agar. Results: We found that cells expressing the phosphomimetic form of Tm1 S283E/Tm1 are characterized by an increased adhesion to the substratum. Moreover, the migration of MDA-MB231/S283E/Tm1 cells in a wound closure assay is reduced compared to parental cells or those expressing the non-phosphorylatable form of Tm1 (S283A). Similarly, the transendothelial migration of MDA-MB231/S283E/Tm1 cells is also reduced as compared to the other cell lines. Moreover, we found that the cells expressing the S283A mutants form more colonies in soft agar that those expressing the S283E mutants. Conclusion: Phosphorylation of Tm1 at Ser283 contributes to its anti-tumor properties, and this effect results mainly from an increase in cell adhesion associated with a decrease in their migratory and invasive potentials.
\end{abstract}

\section{Keywords}

Tropomyosin 1, Phosphorylation, F-Actin, Cell Migration, Invasion

\footnotetext{
${ }^{*}$ Corresponding author.
}

How to cite this paper: Zerradi, M., Houle, F. and Huot, J. (2015) Regulation of Breast Cancer Progression by Phosphorylation of the Tumor Suppressor Tropomyosin-1 Alpha. Journal of Cancer Therapy, 6, 783-792. 


\section{Introduction}

Breast cancer is the most common malignancy among women in Europe and North America. Despite its high incidence, early detection and modern treatments have significantly increased patient survival. Nevertheless, breast cancer remains the second leading cause of cancer death in women [1]. Breast cancer initiation and progression are characterized by multiple genetic and epigenetics alterations that activate dominant-acting oncogenes and disrupt the functions of specific tumor suppressor genes [2]. Genome analyses indicate that there are only a few genes that are commonly mutated in breast cancer. These genes include the oncogens ErbB2, PI3KCA, MYC, and CCND1 as well as the tumor suppressors BRCA1/2 and p53 [2]. PTEN is another important tumor suppressor that counteracts PI3K-mediated survival signaling from activated oncogenes such as ErbB2 [2]. On the other hand, many genes are less frequently mutated, which provides an explanation for the high heterogeneity of breast cancer.

The genes encoding for tropomyosins (Tm) are also frequently deregulated in breast cancer, and significant changes in the expression of different tropomyosin isoforms accompany cell transformation. Mammals have four genes $(\alpha, \beta, \gamma, \delta)$ that code for tropomyosins generating more than 40 alternatively spliced isoforms [3]. The Tm genes have a similar organization. In humans, the $\alpha, \beta, \gamma, \delta$ genes are known as TPM1 (located on the chromosome 15q22) [4], TPM2 (located on chromosome 9p13) [5], TPM3 (located on chromosome 1q22) [6] and TPM4 (located on chromosome 19p13) [7], respectively. Intriguingly, a lot of confusion exists in the nomenclature of the various forms of tropomyosins. In particular, the gene product of TPM2 is Tpm2, TM2 or TM2 and has previously been ascribed as coding for Tm1 in fibroblasts [8] [9]. On the other hand, TPM1 encodes for tropomyosin 1 alpha chain that is also frequently referred as tropomyosin-1 or Tm1 [10]-[12] (www.uniprot.org/uniprot/P09493). For the sake of understanding and to keep in line with previous literature, we used herein TPM2/Tm1 to refer as the gene product of TPM2 and Tm1 as the gene product of TPM1.

The tropomyosins are members of a family of actin-binding proteins that play an important role in regulating the functions of actin filaments in both muscle and non-muscle cells [8] [13]. In striated muscle, tropomyosin regulates the interaction between myosin and actin by forming a troponin complex that mediates muscle contraction in response to calcium [14]. In nonmuscle cells, tropomyosins play a role in the formation and stabilization of stress fibers by facilitating actomyosin interactions and protecting actin against the action of cofilin and gelsolin [14]. The mechanims by which TPM2/Tm1 exerts its tumor suppressor functions in breast cancer seem to be associated with cytoskeletal remodeling. As mentioned above, the TPM2/Tm1 reexpression in MCF7 null cells suppresses the malignant phenotype, which alters the interaction of the E-cadherin-catenin complex with the cytoskeleton, indicating that TPM2/Tm1-induced cytoskeleton dysregulation could play a significant role in suppression of the malignant phenotype [15]. Moreover, the loss of TPM2/Tm1 in cancer cells is associated with disorganization of actin filaments and thereby impaired cell migration [16].

Tropomyosins are subject to two types of posttranslational modification namely, $\mathrm{NH}_{2}$-terminal acetylation and phosphorylation. The acetylation of Tm is essential for normal functions of muscle $\alpha$-Tm and it is required for the strong binding of most Tms to actin [17] [18]. On the other hand, Tm1 phosphorylation modulates its interaction with other proteins required for actin polymerization and stabilization, including caldesmon and HSP27 [19] [20]. Along these lines, the phosphorylation of cytoskeletal non-muscle Tm2 at Ser-61 by phosph-oinositide 3-kinase occurs at a late stage of endocytosis, which is consistent with a role in actin polymerization [21]. In endothelial cells, we found that Tm1 alpha chain (herein named Tm1) is phosphorylated at Ser 283 in response to activation of the ERK/DAP kinase axis by oxidative stress. In turn, this triggers the formation of stress fibers and confers resistance to oxidative stress-mediated membrane blebbing [10]-[12] [22].

In the present study, we investigated the role of phosphorylation in regulating the tumor suppression properties of Tm1 in breast cancer.

\section{Methods}

\subsection{Reagents and Antibodies}

Anti-tropomyosin (clone TM311) monoclonal mouse antibody and anti- $\alpha$-actin antibody produced in rabbit were purchased from Sigma-Aldrich (Oakville, On, Canada). The anti-mouse and anti-rabbit IgG-horseradish peroxidase were purchased from The Jackson Laboratory (Bar Harbor, ME, USA). 


\subsection{Cells}

MDA MB231 cells were obtained from the American Type Culture Collection (ATCC). MDA MB231 cells were maintained in Dulbecco's Modified Eagle Medium (DMED) containing 10\% fetal bovine serum (FBS). Cultures were incubated at $37^{\circ} \mathrm{C}$ in a humidified atmosphere containing 95\% air and 5\% $\mathrm{CO}_{2}$. MDA MB-231 were transfected with vectors expressing wild-type form of Tm1 (Tm1wt), a non phosphorylatable (Tm1 S283A: MDA TM1 A) or a phosphomimetic (Tm1S283E: MDATM1 E) mutated form of Tm1 using the calcium chloride precipitation technique to generate cancer cell lines stably expressing tropomyosin- 1 . The cells were selected by incubation in medium containing G418 at $400 \mu \mathrm{g} / \mathrm{ml}$. A pool of resistant cells was obtained and frozen or sub-cultured for the experiments of this project.

\subsection{Plasmids}

TPM1 (RecName: Full = Tropomyosin alpha-1 chain) cDNA was cloned as previously described (Houle et al. $\mathrm{J}$ Cell Sci 2007) by PCR amplification from IMAGE clone 562592 (ATCC) into pIRES-hrGFP2a (Stratagene, La Jolla, CA) vectors using the following primers:

5'-TAGAATTCTATGGACGCCATCAAGAAGAAGATGCAGATGC-3' and

5'-CCTGCTCGAGTATATGGAAGTCATATCGTTGAGAGC-3'. The tropomyosin-1 Ser 283 Ala was generated by PCR site-directed mutagenesis on pIRES-hrGFP2a-tropomyosin-1 construct using the primers

5'-ATGACTGCTATATAACTCGAGTACCCATATGACG-3' and

5'-TTATATAGCAGTCATATCGTTGAGAGCGTGG-3'.

The tropomyosin-1 S283E mutant was generated similarly using the primers

5' CGATATGACTGAAATAATACTCGAGTACCCATATG-3' and

5’-CGAGTTATATTTCAGTCATATCGTTGAGAGCG-3' (NP_000357) [11].

\subsection{Western Blot}

Total proteins from cells grown in $60 \mathrm{~mm}$ Petri dishes were extracted with $100 \mu \mathrm{l}$ loading buffer. Proteins were quantified using the Lowry method and equal amounts of proteins from each sample were separated on 10\% SDS-PAGE, and the gels were transferred onto nitrocellulose membranes for Western blotting. The membranes were blocked with 5\% non-fat milk in PBS-Tween 20 for one hour at room temperature. Membranes were then incubated over night at $4^{\circ} \mathrm{C}$ in blocking buffer containing 1:1000 dilution of the primary anti-tropomyosin (clone TM311) monoclonal mouse antibodies (Sigma-Aldrich, Oakville, On, Canada). For loading control, 1:3000 dilution of anti- $\alpha$-actin antibody produced in rabbit (Sigma-Aldrich, Oakville, On, Canada) was used. Afterward, membranes were washed and incubated for one hour with the respective horseradish peroxidase-conjugated secondary antibody (anti-IgG antibody) diluted 5000 times. Protein signals were visualised with chemiluminescence Reagent (PerkinElmer, Waltham, MA, USA).

\subsection{Cell Proliferation and Viability Assay}

Cell viability has been determined by using the Quick Cell Proliferation Assay Kit from BioVision. This cell proliferation and viability assay is based on the enzymatic cleavage of the tetrazolium salt WST-1 into formazan dye by cellular mitochondrial dehydrogenases that are active in viable cells only. The formazan dye produced by viable cells is quantified by measuring the absorbance of the dye at $440 \mathrm{~nm}$. The exact procedure that was followed was as described in the BioVision protocol sheet.

\subsection{Cell Adhesion Assays}

MDA MB231-Tm1 cells ( $3 \times 10^{4}$ cells/well) were seeded on plastic in 96 well plates containing DMEM 10\% FBS for 1 hour at $37^{\circ} \mathrm{C}$. Then, the cells were washed twice with fresh DMEM $10 \%$ FBS and fixed with $100 \mu l$ formaldehyde $3.7 \%$ for 30 minutes at room temperature. Thereafter, the cells were washed once with Phosphate Buffered Saline (PBS) and stained with $50 \mu \mathrm{l}$ of crystal violet for $15 \mathrm{~min}$ at room temperature. Then, they were washed twice with PBS and lysed with $100 \mu \mathrm{l}$ of SDS 1\%. Absorbance of each well was measured at $550 \mathrm{~nm}$.

\subsection{Anchorage-Independent Growth Assays}

Liquefied 2\% agarose was mixed with an equal volume of DMEM 2X growth medium lacking serum and sup- 
plemented with G418 (400 ug/ml). One milliliter of the mixture was layered on $35 \mathrm{~mm}$ Petri dishes to create a 1\% agarose base. Liquefied $0.6 \%$ agarose was mixed with an equal volume of DMEM $2 \times$ medium, and $10 \mathrm{ml}$ of this solution was mixed with $1 \mathrm{ml}$ of growth medium containing $10^{4}$ cells in $0.27 \%$ agarose. One $\mathrm{ml}$ of this cell suspension was layered on top of the $1 \%$ agarose base, and $1 \mathrm{ml}$ of DMEM medium containing $10 \%$ FBS was added. The cells were incubated for 14 days, after which colonies present in representative fields were photographed using phase-contrast microscopy (20× and 40× on a Nikon-TE300 inverted microscope) [23].

\subsection{Cell Migration Assays}

Cell migration was evaluated by using two methods:

\subsubsection{Wound Healing Assay}

MDA MB231, MDA MB231/Tm1wt, MDA MB231/Tm1 S283A and MDA MB231/Tm1 S283E were cultivated in complete growth medium. Cells were seeded at high density in $35 \mathrm{~mm}$ Petri dishes in complete growth medium. One day later, a straight scratch was done using a p200 micropipette tip. Thereafter, cells were washed three times with fresh DMEM and incubated in the same medium. The movements of cells in the scratched area were monitored by capturing images every 15 minutes for a total duration of $24 \mathrm{~h}$ using a $10 \times$ objective lens of a phase contrast microscope (TE2000, Nikon). Then, migrated cells were counted manually.

\subsubsection{Transendothelial Cell Migration Assays}

Cell migration was assayed using a modified Boyden chamber assay. HUVEC $\left(150 \times 10^{3}\right)$ were grown to confluence $(48 \mathrm{~h})$ on an $8.0 \mathrm{~m}$ pore size gelatinized polycarbonate membranes (Corning, NY, USA) separating the two compartments of a $6.5 \mathrm{~mm}$ Transwell. Tumor cells in suspension were stained with $500 \mathrm{nM}$ calcein-AM (Sigma-Aldrich, Oakville, On, Canada) for $30 \mathrm{~min}$ at $37^{\circ} \mathrm{C}$, and then added in migration buffer (199 medium, 10 mM HEPES pH 7.4, $1 \mathrm{mM} \mathrm{MgCl}_{2}$, and $0.5 \%$ bovine serum albumin) on the endothelial monolayer. The chambers were incubated for $6 \mathrm{~h}$ at $37^{\circ} \mathrm{C}$ in a $5 \% \mathrm{CO}_{2}$ atmosphere. The cells in the upper part of the chamber were removed with a cotton swab. Then, fluorescent tumor cells that crossed the membrane were counted in five fields using a 20× lens on a Nikon-TE300 inverted microscope [24].

\subsection{Statistics}

Values are expressed as mean \pm SD. Student $t$ tests were used for comparison between two means. A $p$ value $<0.05$ was considered as statistically significant.

\section{Results}

\subsection{Adhesion of MDA MB231 Cells Depends on Phosphorylation of Tm1 at Ser 283}

Different isoforms of tropomyosins have been shown to exert tumor suppressor properties in breast cancer cells [8]. Along these lines, parental MDA MB231 cells are highly invasive and they do not express Tm1 (Figure 1) and [25]. Accordingly, they proliferate faster than pooled clonal MDA MB231 cell lines expressing a wt form of Tm1 (Figure 2). However, the mechanisms underlying the tumor suppressors functions of Tm1 are still unclear. We reported previously that Tm1 is phosphorylated at S283 in endothelial cells exposed to oxidative stress, which contributes to maintain the integrity of the endothelium [11] [12] [22]. Here, we hypothesize that phosphorylation of Tm1 at S283 contributes to regulate its tumor suppressor functions. To this end, we generated clonal MDA MB231 breast cancer cells that stably express non-phosphorylatable (S283A) or phosphomimetic (S283E) mutated forms of the protein (Figure 1). Thereafter, we pooled the clonal cell lines and used them to investigate the role of $\mathrm{Tm} 1$ phosphorylation in modulating its function of tumor suppressor.

We first verified whether the phospohorylation of Tm1 regulates cellular adhesion by means of adhesion assays performed directly on plastic dishes. Results showed that the cells that express the S283E/Tm1 phosphomimetic mutant were those that adhere more strongly to the substratum either after $1 \mathrm{~h}$ and $2 \mathrm{~h}$. Conversely, the parental cells and the cells that express the non-phosphorylatable mutant S283A/Tm1 were those that adhere the less (Figure 3). Overall, these findings suggest that phosphorylation of Tm1 at S283 increased the adhesion of MDA231 cells. Interestingly, consistent with the fact that MDA MB231/S283E/Tm1 has a higher adhesive ability than the two other cell lines, we previously reported that these cells express thick stress fibers whereas 


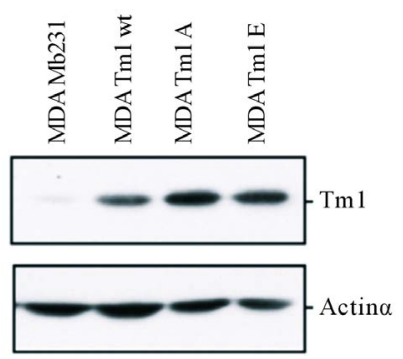

Figure 1. Generation of MDA MB-231 cells stably expressing wild type forms of Tm1 or phosphomimetic or nonphosphorylatable forms of Tm1. MDA MB-231 were transfected with vectors expressing wild-type form of Tm1 (Tm1wt), a non phosphorylatable (Tm1 S283A: MDA TM1 A) or a phosphomimetic (Tm1S283E: MDATM1 E) mutated form of $\operatorname{Tm} 1$ using the calcium chloride precipitation technique. The cells were selected by incubation in medium containing G418 at $400 \mu \mathrm{g} / \mathrm{ml}$. A pool of resistant cells were obtained and used in experiments described in the paper. Western blot was carried out with proteins extracted from the cell lines expressing the various forms of Tm1, using tropomyosin antibody.

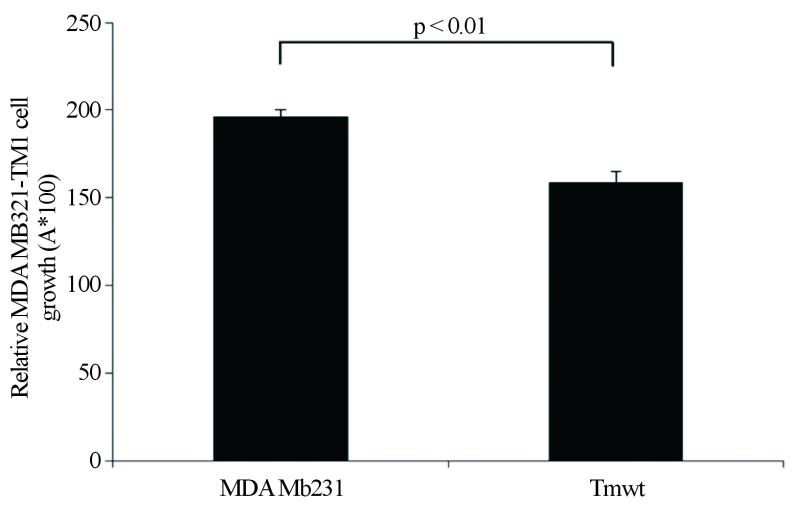

Figure 2. Tropomyosin-1 expression in MDA MB231 cell lines reduces their proliferation. Parental MDA MB231 cells and MDA MB231 expressing wt Tm1 (TMwt) were incubated with medium supplemented with 10\% FBS for $96 \mathrm{~h}$. Cell proliferation was determined using a colorimetric method based on conversion of tetrazolium salt WST-1 to a colored compound. Values are average \pm SD from an experiment done in triplicates. P value was determined by a Student's t-test. The experiment was performed at least three times in triplicates.

parental MDA MB231 cells as well as MDA MB231/S283A/Tm1 cells are devoid of stress fibers [11]. Indeed, stress fibers are well recognized to be associated with increased cellular adhesion [26] [27].

\subsection{Phosphorylation of Tm1 at S283 Reduced Cell Migration}

Increased cell migration is an inherent feature that characterized invasiveness during metastatic dissemination [28]. We thus evaluated next whether phosphorylation of Tm1 at S283 regulates cell migration by means of a wound-healing assay applied to the different MDA MB231 cell lines. We found that the migration potential of MDA MB231/S283E/Tm1 cells was reduced as compared to parental cells that do not express Tm1 or to the MDA 231/S283A/Tm1 cells. This is evidenced by the larger number of cells from the latter two cell lines that invade the scratch (Figure 4(a), Figure 4(b)). These findings suggest that phosphorylation of Tm1 at S283 reduced the invasive properties of metastatic MDA MB231 cells. Incidentally, these results are in line with the fact that Tm1 phosphorylation at S283 has conferred increased adhesion of the cells to the substratum rendering 


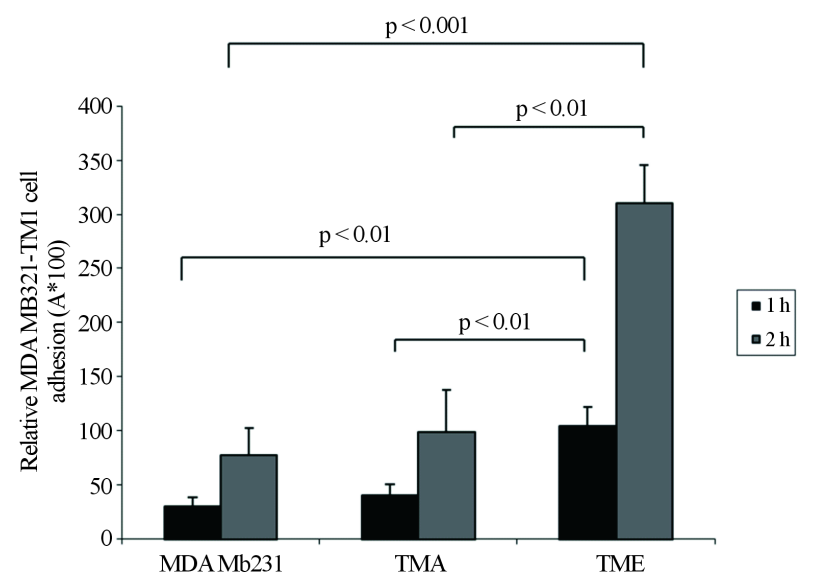

Figure 3. Phosphorylation of Tm1 at S283 increases their adhesion to substratum. Parental MDA MB231-Tm1 or MDA MB-231 cells stably expressing non phosphorylatable (Tm1S283A: TMA) or a phosphomimetic (Tm1S283E: TME) were incubated with medium supplemented with $10 \%$ FBS for $1-2$ hours at $37^{\circ} \mathrm{C}$. Thereafter, the cells were fixed with formaldehyde 3.7\%, washed with PBS, stained with crystal violet and lysed with SDS $1 \%$. Absorbance was used as the measure of adhesion. Values are average \pm SD from an experiment done in triplicates. The experiments were performed at least three times in triplicates. $\mathrm{p}$ value was determined by a Student's t-test.

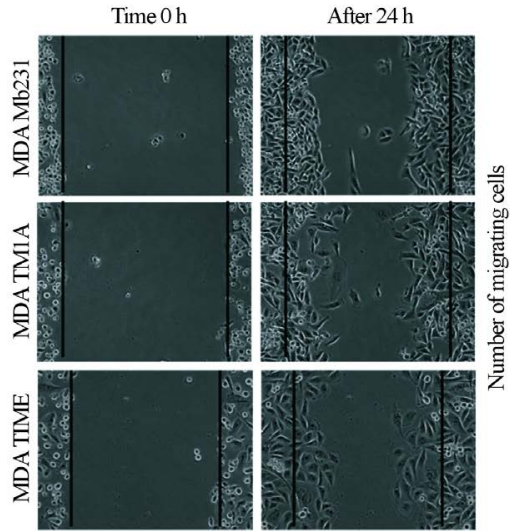

(a)

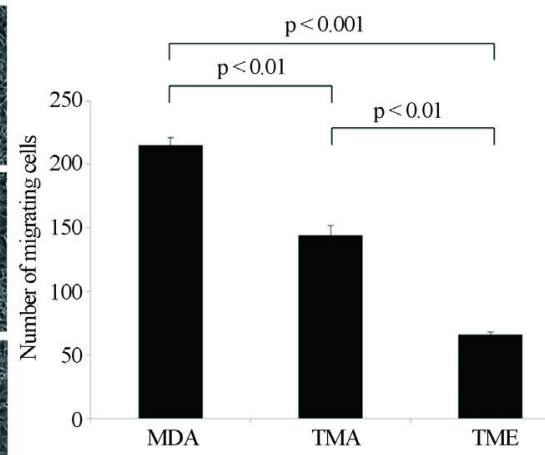

(b)

Figure 4. Phosphorylation of Tm1 at S283 reduces cell migration in MDA MB231. (a) Cell migration was evaluated by means of a scratch test assay applied to parental MDA MB231 and MDA MB231 cells stably expressing Tm1S283A (TMA) or Tm1S283E (TME). The black lines represent the initial wound and were used to visualize the cells that invaded the scratch. (b) The cells that crossed the lines were manually counted from four different fields. Their average number \pm SD from two separate experiments are indicated in ordinate. $\mathrm{p}$ value was determined by a Student's t-test.

them less motile.

Transendothelial migration and extravasation of circulating cancer cells are key events of metastatic dissemination [29] [30]. Here we found that the transendothelial migration of MDA MB231/S283E/Tm1 cells was reduced in comparison to the other cell lines (Figure 5). This result indicates that the phosphorylation of Tm1 impairs the migration of MDA MB231 through endothelial layer.

Overall, this series of results indicate that phosphorylation of Tm1 at S283 reduces the migration of MDA MB231 cells and thereby their metastatic potential, which is consistent with the fact that phoshorylation of Tm1 regulates its functions of tumor suppressor. 


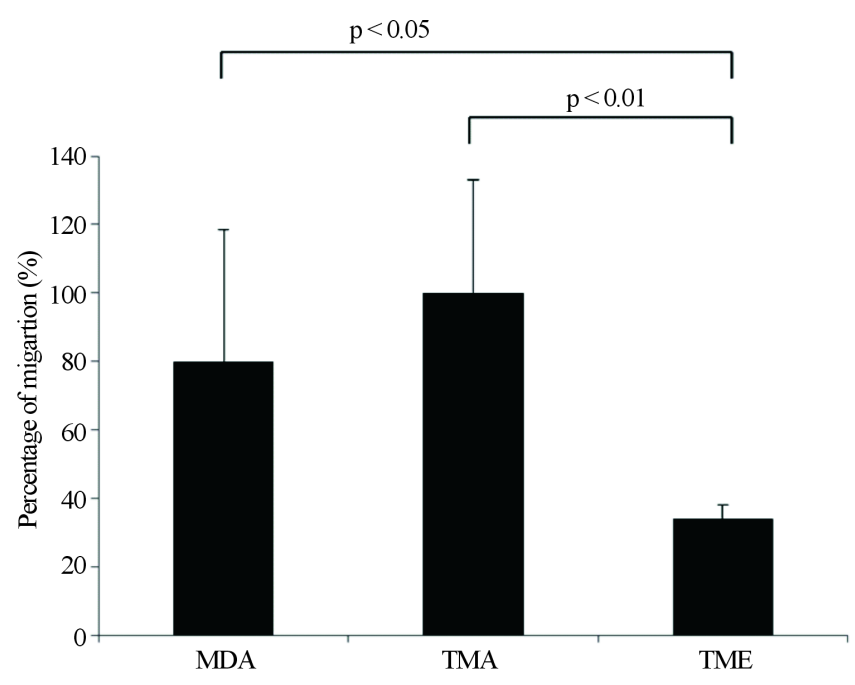

Figure 5. Phosphorylation of Tm1 at S283 reduces transendothelial cell migration of MDA MB231. Transendothelial cell migration of parental MDA MDA MB231 and MDA MB231 cells stably expressing Tm1S283A (TMA) or Tm1S283E (TME). Briefly, HUVEC $\left(150 \times 10^{3}\right)$ were grown to confluence $(48 \mathrm{~h})$ on a $8.0 \mathrm{~m}$ pore size gelatinized polycarbonate membranes separating the two compartments of a $6.5 \mathrm{~mm}$ Transwell. MDA MB231 cells in suspension were stained with $500 \mathrm{nM}$ calcein- $\mathrm{AM}$ for $30 \mathrm{~min}$ at $37^{\circ} \mathrm{C}$, and then added in migration buffer on the endothelial monolayer. The chambers were incubated for $6 \mathrm{~h}$ at $37^{\circ} \mathrm{C}$ in a $5 \% \mathrm{CO}_{2}$ atmosphere. The cells in the upper part of the chamber were removed with a cotton swab. Then, fluorescent MDA MB231 cells that crossed the membrane were counted in five fields using a 20× lens on a Nikon-TE300 inverted microscope [24]. Values are average \pm SD from two independent experiments done in triplicates. P value was determined by a Student's t-test.

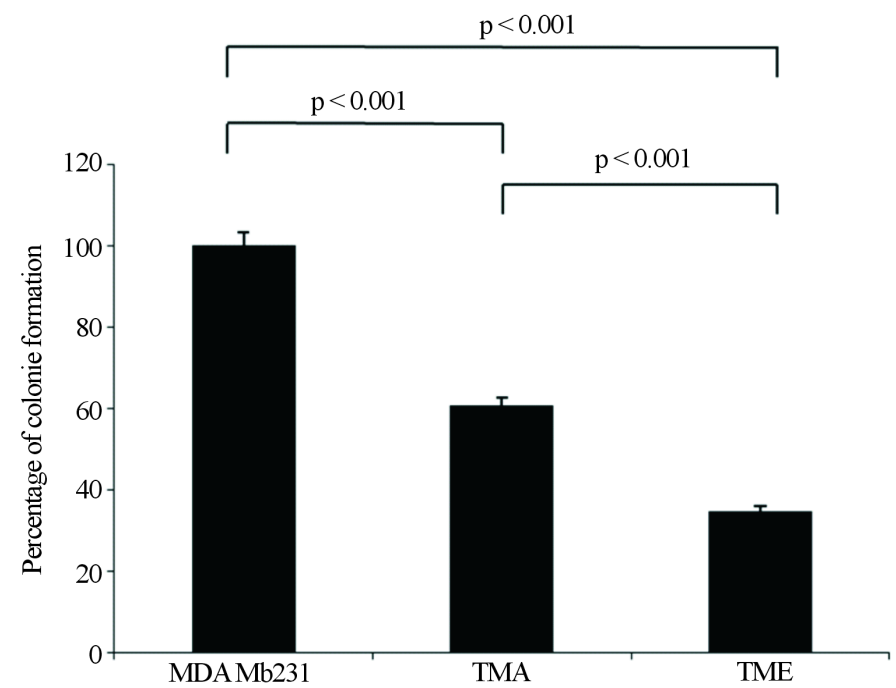

Figure 6. Phosphorylation of Tm1 at S283 reduces the formation of MDAMB231 colonies on soft agar by cells. Liquefied $0.6 \%$ agarose was mixed with an equal volume of DMEM $2 \times$ medium. Ten $\mathrm{ml}$ of this solution was mixed with 1 $\mathrm{ml}$ of growth medium containing $10^{4}$ cells in $0.27 \%$ agarose. One $\mathrm{ml}$ of this cell suspension was layered on top of $1 \%$ agarose base, and $1 \mathrm{ml}$ of DMEM medium containing 10\% FBS was added. The cells (parental MDA MB231, MDA MB231 S283A (TMA) or MDA MB231S283E (TME) were incubated for 14 days, after which colonies present in 5 representative fields were counted. P value was determined by a Student's t-test. 


\subsection{Phosphorylation of Tm1 at S283 Reduces the Formation of MDA MB231 Colonies on Soft Agar}

The anchorage-independent growth assay for colony formation in soft agar, is a stringent assay for detecting malignant transformation of cells in vitro and is considered as a hallmark of malignant transformation [31]. We thus used this assay as an additional end-point to evaluate the role of $\operatorname{Tm} 1$ phosphorylation as a regulatory mechanism underlying the tumor suppressor properties of $\mathrm{Tm} 1$ in breast cancer. As shown in Figure 6, the expression of the S283E mutant reduced by $70 \%$ the number of colonies formed by the parental MDA MB231 cells in soft agar. In contrast, the formation of colonies by cells expressing Ser283A was twice higher than those expressing the S283E mutant. The results indicate that phosphorylation of $\operatorname{Tm} 1$ at Ser283 reduces the anchorage-independent growth of MDA MB231 cells, which is consistent with the fact that phosphorylation of Tm1 contributes to its tumor suppressor properties.

\section{Discussion}

High-molecular weight tropomyosins such as Tm1 play major roles as tumor suppressor in breast cancer [13] [32]. However, the mechanisms underlying the tumor suppressor properties of Tm1 are ill defined. Here we provided the first evidence showing that phoshorylation of Tm1 is a major mechanism that regulates its tumor suppressor functions. This is supported first by the observation showing that MDA MB231 cells that express the phosphomimetic mutant S283E adhere more strongly to the substratum than the parental cells and those that express the non-phosphorylatable mutant S283A. Indeed, this finding is consistent with the fact that MDA MB231/ S283E/Tm1 are less motile and invasive given that are sticked to the substratum, an effect explained by the fact that MDA MB231/S283E/Tm1 cells contains more stress fibers [11]. Along these lines, a second argument supporting the role of phosphorylation of Tm1 at Ser283 in mediating its tumor suppressor functions is that MDA MB231/S283E/Tm1 are less motile than the parental cells and the cells that express the non-phosphorylatable mutant of Tm1 in the wound closure assay. Indeed, it is well known that increased invasive properties are associated with increased cell migration [28]. Third, MDA MB231/S283E/Tm1 cells have a lower capacity to transmigrate across an endothelial layer than the parental cells and the cells that express the non-phosphorylatable mutant of Tm1. This observation is a strong argument favoring the point that phosphorylation of Tm1 at S283 is involved in conferring tumor suppression and antimetastatic potential to Tm1 given that TEM is a well-known prerequesite to metasasise [29]. We previously reported that TEM of colon cancer cells require interactions between adhesion receptors present on endothelial cells and their counter-receptors on cancer cells. We showed that E-selectin is a typical endothelial adhesion receptor that is induced by inflammatory cytokines and that binds to Death Receptor 3 expressed by colon cancer cells to enable their transendothelial migration and extravasation [24] [29] [33]. However, it does not seem that the TEM of MDA MB231 cells do require the expression of E-selectin given that they cross the endothelial layer even when endothelial cells do not express E-selectin. Hence, in the present study, our results suggest that Tm1-dependent TEM of MDA MB231 cells is importantly dependent on its phosphorylation at Ser283. Finally, the fact that cells expressing the phosphomimetic mutant of Tm1 form less colonies in soft agar is a major argument supporting that Tm1 phosphoryation at Ser 283 confers tumor suppressor functions to Tm1, given that this test is well accepted as reflecting malignancy in vitro [31]. Nevertheless, the finding that the cells expressing S283A mutant also form less colonies than the parental cells, but more than the S283E, cells indicate that other factors than just phosphorylation are involved in tumor suppression by Tm1.

Overall our results constitute the first evidence that phosphorylation of Tm1 regulates its tumor suppressor functions in breast cancer cells.

\section{Conclusion}

In this study, we have shown that MDA-MB231 cells stably express a pseudophosphorylated form of Tm1 at Ser283 (S283E) adhere more firmly to the substratum and are less motile that the parental cells and cells that express a non-phosphorylatable form of the protein (S283A). Moreover, the Tm1 S283E expressing MDA-231 cells form fewer than the TM1 S283A expressing cells colonies in soft agar. We thus conclude that phosphoryation of Tm1 at Ser283 is a major mechanism that contributes to the tumor and metastatic suppressor properties of Tm1.

\section{Acknowledgements}

This work was supported by the Canadian Institutes of Health Research. 


\section{Competing Interests}

The authors declare that they have no competing interests.

\section{Authors' Contributions}

MZ and JH designed the study. MZ and FH carried out the experimental studies. MZ, JH and FH prepared the manuscript. All authors read and approved the final manuscript.

\section{References}

[1] Hooks, M.A. (2010) Breast Cancer: Risk Assessment and Prevention. Southern Medical Journal, 103, 333-338. http://dx.doi.org/10.1097/SMJ.0b013e3181d39038

[2] Lee, E.Y. and Muller, W.J. (2010) Oncogenes and Tumor Suppressor Genes. Cold Spring Harbor Perspectives in Biology, 2, a003236. http://dx.doi.org/10.1101/cshperspect.a003236

[3] Perry, S.V. (2001) Vertebrate Tropomyosin: Distribution, Properties and Function. Journal of Muscle Research \& Cell Motility, 22, 5-49. http://dx.doi.org/10.1023/A:1010303732441

[4] Eyre, H., Akkari, P.A., Wilton, S.D., Callen, D.C., Baker, E. and Laing, N.G. (1995) Assignment of the Human Skeletal Muscle Alpha-Tropomyosin Gene (TPM1) to Band 15q22 by Fluorescence in Situ Hybridization. Cytogenetics and Cell Genetics, 69, 15-17. http://dx.doi.org/10.1159/000133928

[5] Hunt, C.C., Eyre, H.J., Akkari, P.A., Meredith, C., Dorosz, S.M., Wilton, S.D., Callen, D.F., Laing, N.G. and Baker, E. (1995) Assignment of the Human Beta Tropomyosin Gene (TPM2) to Band 9p13 by Fluorescence in Situ Hybridisation. Cytogenetics and Cell Genetics, 71, 94-95. http://dx.doi.org/10.1159/000134070

[6] Wilton, S.D., Eyre, H., Akkari, P.A., Watkins, H.C., MacRae, C., Laing, N.G. and Callen, D.C. (1995) Assignment of the Human $\alpha$-Tropomyosin Gene TPM3 to $1 \mathrm{q} 22 \rightarrow \mathrm{q} 23$ by Fluorescence in Situ Hybridisation. Cytogenetics and Cell Genetics, 68, 122-124. http://dx.doi.org/10.1159/000133905

[7] Wilton, S.D., Lim, L., Dorosz, S.D., Gunn, H.C., Eyre, H.J., Callen, D.F. and Laing, N.G. (1996) Assignment of the Human Alpha-Tropomyosin Gene TPM4 to Band 19p13.1 by Fluorescence in Situ Hybridization. Cytogenetics and Cell Genetics, 72, 294-296. http://dx.doi.org/10.1159/000134206

[8] Gunning, P., O’Neill, G. and Hardeman, E. (2008) Tropomyosin-Based Regulation of the Actin Cytoskeleton in Time and Space. Physiological Reviews, 88, 1-35. http://dx.doi.org/10.1152/physrev.00001.2007

[9] Geeves, M.A., Hitchcock-DeGregori, S.E. and Gunning, P.W. (2015) A Systematic Nomenclature for Mammalian Tropomyosin Isoforms. Journal of Muscle Research and Cell Motility, 36, 147-153.

[10] Simoneau, B., Houle, F. and Huot, J. (2012) Regulation of Endothelial Permeability and Transendothelial Migration of Cancer Cells by Tropomyosin-1 Phosphorylation. Vascular Cell, 4, 18. http://dx.doi.org/10.1186/2045-824x-4-18

[11] Houle, F., Poirier, A., Dumaresq, J. and Huot, J. (2007) DAP Kinase Mediates the Phosphorylation of Tropomyosin-1 Downstream of the ERK Pathway, Which Regulates the Formation of Stress Fibers in Response to Oxidative Stress. Journal of Cell Science, 120, 3666-3677. http://dx.doi.org/10.1242/jcs.003251

[12] Houle, F., Rousseau, S., Morrice, N., Luc, M., Mongrain, S., Turner, C.E., Tanaka, S., Moreau, P. and Huot, J. (2003) Extracellular Signal-Regulated Kinase Mediates Phosphorylation of Tropomyosin-1 to Promote Cytoskeleton Remodeling in Response to Oxidative Stress: Impact on Membrane Blebbing. Molecular Biology of the Cell, 14, 1418-1432. http://dx.doi.org/10.1091/mbc.E02-04-0235

[13] Gunning, P. (2008) Emerging Issues for Tropomyosin Structure, Regulation, Function and Pathology. Advances in Experimental Medicine and Biology, 644, 293-298. http://dx.doi.org/10.1007/978-0-387-85766-4_22

[14] Cooper, J. (2002) Actin Dynamics: Tropomyosin Provides Stability. Current Biology, 12, R523-R525. http://dx.doi.org/10.1016/s0960-9822(02)01028-X

[15] Mahadev, K., Raval, G., Bharadwaj, S., Willingham, M.C., Lange, E.M., Vonderhaar, B., Salomon, D. and Prasad, G.L. (2002) Suppression of the Transformed Phenotype of Breast Cancer by Tropomyosin-1. Experimental Cell Research, 279, 40-51. http://dx.doi.org/10.1006/excr.2002.5583

[16] Hendricks, M. and Weintraub, H. (1981) Tropomyosin Is Decreased in Transformed Cells. Proceedings of the National Academy of Sciences of the United States of America, 78, 5633-5637. http://dx.doi.org/10.1073/pnas.78.9.5633

[17] Urbancikova, M. and Hitchcock-DeGregori, S.E. (1994) Requirement of Amino-Terminal Modification for Striated Muscle Alpha-Tropomyosin Function. The Journal of Biological Chemistry, 269, 24310-24315.

[18] Pittenger, M.F., Kistler, A. and Helfman, D.M. (1995) Alternatively Spliced Exons of the Beta Tropomyosin Gene Exhibit Different Affinities for F-Actin and Effects with Nonmuscle Caldesmon. Journal of Cell Science, 108, 3253-3265. 
[19] Somara, S., Pang, H. and Bitar, K.N. (2005) Agonist-Induced Association of Tropomyosin with Protein Kinase Calpha in Colonic Smooth Muscle. American Journal of Physiology: Gastrointestinal and Liver Physiology, 288, G268-G276. http://dx.doi.org/10.1152/ajpgi.00330.2004

[20] Mak, A., Smillie, L.B. and Barany, M. (1978) Specific Phosphorylation at Serine-283 of Alpha Tropomyosin from Frog Skeletal and Rabbit Skeletal and Cardiac Muscle. Proceedings of the National Academy of Sciences of the United States of America, 75, 3588-3592. http://dx.doi.org/10.1073/pnas.75.8.3588

[21] Naga Prasad, S.V., Jayatilleke, A., Madamanchi, A. and Rockman, H.A. (2005) Protein Kinase Activity of Phosphoinositide 3-Kinase Regulates Beta-Adrenergic Receptor Endocytosis. Nature Cell Biology, 7, 785-796. http://dx.doi.org/10.1038/ncb1278

[22] Houle, F. and Huot, J. (2006) Dysregulation of the Endothelial Cellular Response to Oxidative Stress in Cancer. Molecular Carcinogenesis, 45, 362-367. http://dx.doi.org/10.1002/mc.20218

[23] Desprez, P.Y., Lin, C.Q., Thomasset, N., Sympson, C.J., Bissell, M.J. and Campisi, J. (1998) A Novel Pathway for Mammary Epithelial Cell Invasion Induced by the Helix-Loop-Helix Protein Id-1. Molecular and Cellular Biology, 18, 4577-4588.

[24] Gout, S., Morin, C., Houle, F. and Huot, J. (2006) Death Receptor-3, a New E-Selectin Counter-Receptor That Confers Migration and Survival Advantages to Colon Carcinoma Cells by Triggering p38 and ERK MAPK Activation. Cancer Research, 66, 9117-9124. http://dx.doi.org/10.1158/0008-5472.CAN-05-4605

[25] Yu, M., Sato, H., Seiki, M. and Thompson, E.W. (1995) Complex Regulation of Membrane-Type Matrix Metalloproteinase Expression and Matrix Metalloproteinase-2 Activation by Concanavalin A in MDA-MB-231 Human Breast Cancer Cells. Cancer Research, 55, 3272-3277.

[26] Kirchenbuchler, D., Born, S., Kirchgessner, N., Houben, S., Hoffmann, B. and Merkel, R. (2010) Substrate, Focal Adhesions, and Actin Filaments: A Mechanical Unit with a Weak Spot for Mechanosensitive Proteins. Journal of Physics: Condensed Matter, 22, Article ID: 194109. http://dx.doi.org/10.1088/0953-8984/22/19/194109

[27] O’Neill, G.M. (2009) The Coordination between Actin Filaments and Adhesion in Mesenchymal Migration. Cell Adhesion \& Migration, 3, 355-357. http://dx.doi.org/10.4161/cam.3.4.9468

[28] Geho, D.H., Bandle, R.W., Clair, T. and Liotta, L.A. (2005) Physiological Mechanisms of Tumor-Cell Invasion and Migration. Physiology (Bethesda), 20, 194-200. http://dx.doi.org/10.1152/physiol.00009.2005

[29] Gout, S., Tremblay, P.L. and Huot, J. (2008) Selectins and Selectin Ligands in Extravasation of Cancer Cells and Organ Selectivity of Metastasis. Clinical \& Experimental Metastasis, 25, 335-344. http://dx.doi.org/10.1007/s10585-007-9096-4

[30] Gout, S. and Huot, J. (2009) Role of Cancer Microenvironmentin Metastasis: Focus on Colon Cancer. Cancer Microenvironment, 1, 69-83. http://dx.doi.org/10.1007/s12307-008-0007-2

[31] Mori, S., Chang, J.T., Andrechek, E.R., Matsumura, N., Baba, T., Yao, G., Kim, J.W., Gatza, M., Murphy, S. and Nevins, J.R. (2009) Anchorage-Independent Cell Growth Signature Identifies Tumors with Metastatic Potential. Oncogene, 28, 2796-2805. http://dx.doi.org/10.1038/onc.2009.139

[32] Shah, V., Braverman, R. and Prasad, G.L. (1998) Suppression of Neoplastic Transformation and Regulation of Cytoskeleton by Tropomyosins. Somatic Cell and Molecular Genetics, 24, 273-280. http://dx.doi.org/10.1023/B:SCAM.0000007130.08611.fC

[33] Tremblay, P.L., Huot, J. and Auger, F.A. (2008) Mechanisms by Which E-Selectin Regulates Diapedesis of Colon Cancer Cells under Flow Conditions. Cancer Research, 68, 5167-5176. http://dx.doi.org/10.1158/0008-5472.CAN-08-1229

\section{List of Abbreviations}

Tm1: $\quad$ Tropomyosin 1 alpha chain;

Tm1 S283A: non phosphorylatable mutated form of Tm1;

Tm1S283E: $\quad$ phosphomimetic mutated form of Tm1;

DMED: $\quad$ Dulbecco's Modified Eagle Medium;

FBS: $\quad$ fetal bovine serum;

PBS: $\quad$ phosphate-buffered saline;

PBS-T: $\quad$ PBS-tween 20;

SDS-PAGE: $\quad$ sodium dodecyl sulphate-polyacrylamide gel electrophoresis;

WT: $\quad$ wild type. 